\title{
SAMBA: Superconducting Antenna-coupled, Multi-frequency, Bolometric Array
}

\author{
Alexey Goldin*, James J. Bock*, Cynthia Hunt ${ }^{\dagger}$, Andrew E. Lange ${ }^{\dagger}$, \\ Henry LeDuc*, Anastasios Vayonakis ${ }^{\dagger}$ and Jonas Zmuidzinas ${ }^{\dagger}$ \\ ${ }^{*} J P L, 4800$ Oak Grove drive, Pasadena, CA \\ ${ }^{\dagger}$ California Institute of Technology, Pasadena, CA
}

\begin{abstract}
We present a design for a multipixel, multiband $(100 \mathrm{GHz}, 200 \mathrm{GHz}$ and $400 \mathrm{GHz})$ submillimeter instrument: SAMBA (Superconducting Antenna-coupled, Multi-frequency, Bolometric Array). SAMBA uses slot antenna coupled bolometers and microstrip filters. The concept allows for a much more compact, multiband imager compared to a comparable feedhorn-coupled bolometric system. SAMBA incorporates an array of slot antennas, superconducting transmission lines, a wide band multiplexer and superconducting transition edge bolometers. The transition-edge film measures the millimeter-wave power deposited in the resistor that terminates the transmission line.
\end{abstract}

\section{INTRODUCTION}

The SAMBA architecture promises a lot of advantages compared to existing systems. Unlike phase sensitive detection techniques, direct detectors can be photon-noise limited under extremely low-backgrounds, even those of dispersive spectroscopy with a cooled aperture. Antenna-coupled bolometers provide additional systems advantages such as greatly reduced thermally active area, volume, heat capacity and thermal conductivity, and improved rejection of stray light and out-of-band radiation. The large sub-K feedhorn optics and metal-mesh filters currently required by missions such as Hershel and FIRST which comprise $>95 \%$ of the sub-K focal plane mass and volume would be eliminated by the new focal plane architectures.

To realize these advantages, we need to refine and combine the following existing technologies: wideband superconducting stripline filters, long superconducting transmission lines, and a large (for this band) area synthesized phased array using slot antennae.

\section{FILTERS}

To design a frequency multiplexor, we needed fairly wideband filters with high out-ofband input impedance. Using the prototype from [1], we designed a lumped elements prototype $30 \%$ wide Chebyshev three pole $0.5 \mathrm{db}$ filter (figure 1). Then capacitors and $L C$ elements were approximated by radial stubs and a combination of coplanar waveguide with microstrip and analyzed with Supermix[2] - a C++ library, developed

CP605, Low Temperature Detectors, edited by F. S. Porter et al. (C) 2002 American Institute of Physics 0-7354-0049-0/02/\$19.00 251 


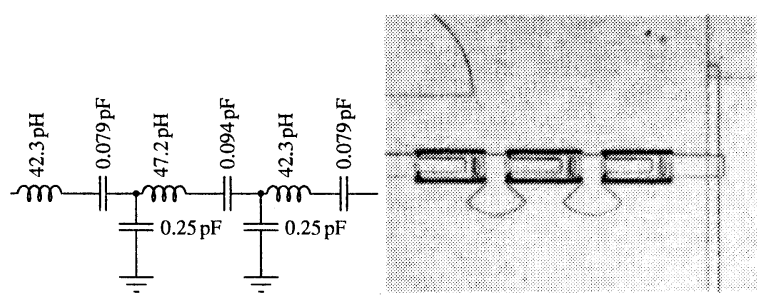

FIGURE 1. Filter schematics. Center frequency is $100 \mathrm{GHz}$, transmission line impedance $-5 \Omega$. On the right: $350 \mathrm{GHz}$ band filter manufactured at Micro Devices Laboratory (MDL) at JPL.
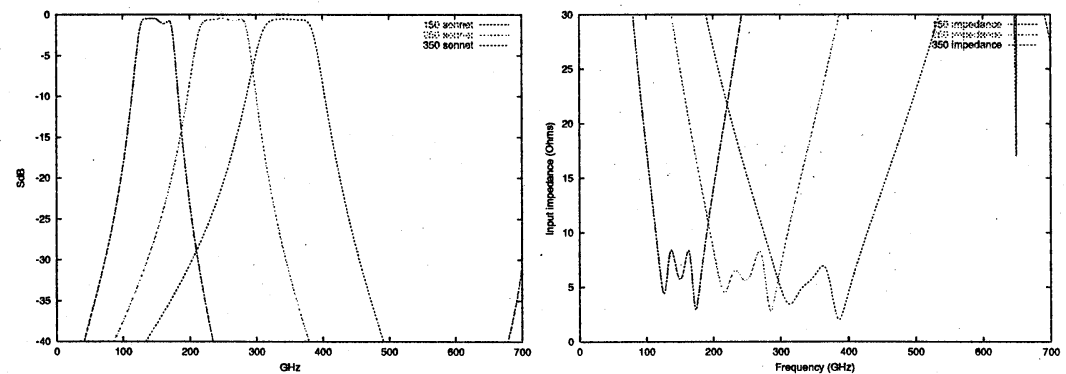

FIGURE 2. On the left: simulated transmission of three filters with bandpasses centered at 150,250 and $350 \mathrm{GHz}$. These are not the same bands as required for antenna in figure 3. Because of small (compared to wavelength) size of filter elements, the next resonance of $150 \mathrm{GHz}$ filter does not start until well beyond the range of highest frequency filter, where it can be easily suppressed by lowpass filter if necessary. On the right impedances of all 3 filters are shown. Out of band impedances are high as needed for multiplexor.

in Caltech primarily for simulation of SIS mixers. To finalize design, we used numerical simulation with well-known Sonnet package.

\section{FOCAL PLANE ANTENNA}

The SAMBA architecture is using a passive phased array antenna. A familiar analog of the SAMBA is a phased array of transmitting antennas. The amplitude and phase of the electric field at each antenna control the pointing and far-field beam pattern of such a phased array. Rather than actively controlling the electric field of each antenna, SAMBA operates by passively adding the electric fields of each antenna in a phasecoherent manner to synthesize diffraction-limited beams at multiple frequencies.

The fundamental element of such an array is the long slot antenna cut into a niobium ground plane. Our numerical studies of such an array demonstrate fairly good broadband performance. The focal plane structure consists of slots separated by $d$, and every slot tapped with a step $d$. One of the restrictions for $d$ is the following: $d<\lambda_{\min } / 2$, where $\lambda_{\min }$ is the shortest wavelength of interest in silicon (to avoid scattering radiation into sidelobes and surface waves). Another requirement is to maintain real part of antenna 

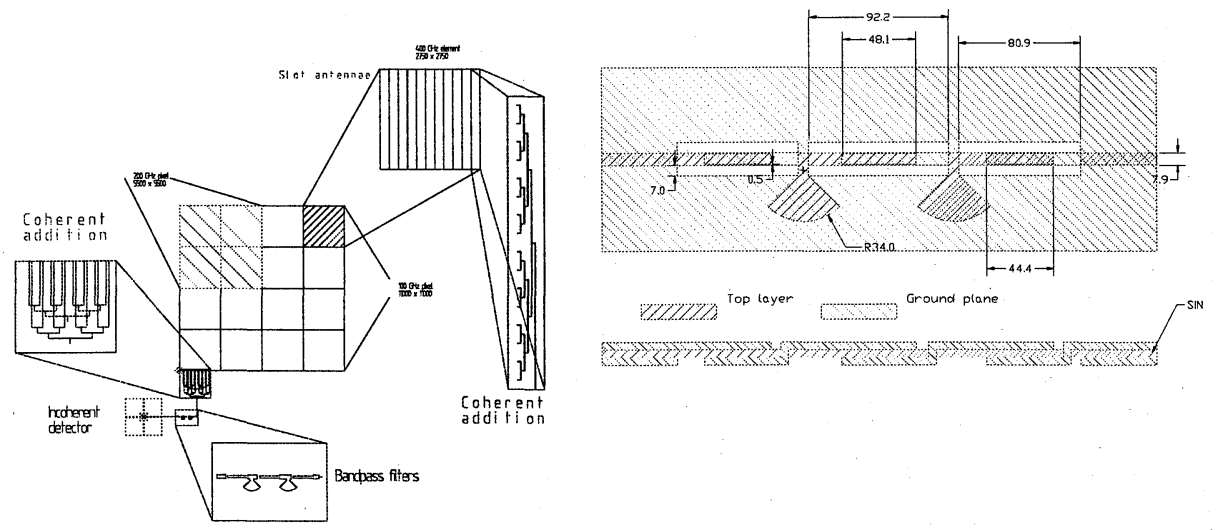

FIGURE 3. SAMBA integrated focal plane consisting of a densely packed array of slot antennas in a Nb ground plane deposited on silicon. Microstrip taps cross the slot antennae and are coherently summed, first along columns (right top inset), then along rows to a single transmission line per Airy disk at $400 \mathrm{GHz}$, tapered to impedance of $5 \Omega$. The line passes through a bank of filters. Low frequency lines are coherently summed again, forming virtual diffraction size limited low frequency detectors. Then microstrip line goes through a filter bank and terminates at normal film resistor, placed on SiN insulated island. The resistor temperature is read out by TES thermistor, located at the same island. On the right: design of a $100 \mathrm{GHz}$ filter. Both groundplane and microstrip layer are 3000 Åniobium, with penetration depth assumed to be about $700 \AA$. Dielectric between two layers of niobium $-2000 \AA$ of silicon oxide. The whole structure is photolitographed on silicon wafer.

impedance high enough to assure good coupling from transmission line to antenna. In our case this second requirement was more restrictive, setting $d$ to no more then $100 \mu \mathrm{m}$.

The total size of the array is dictated by the size of Airy pattern at lowest frequency. In our case, for $F / 3.3$ optical system, the total array size is $11000 \mu \mathrm{m}$ by $11000 \mu \mathrm{m}$.

We have done extensive EM simulations of the antenna array using our own software which generalizes method described in [3]. The program is written in Numerical Python and scales well up to arrays of 16 by 16 antennas on $450 \mathrm{MHz}$ Pentium III desktop computer. We also wrote another program to simulate infinite periodic array of slots. The output of these programs is antenna impedance and voltage distributions along the slot, which can be used to calculate beammap. Both of these programs give very similar results for impedance of the antenna and beamshape.

\section{LOSSES}

The major sources of inefficiency are losses in transmission lines (see poster M03 by Anastasios Vayonakis), dielectric losses (unwanted radiation into lower hemisphere and reflection at dielectric interfaces) and losses due to impedance mismatch between antenna and transmission line. Transmission line losses will be highest (probably at least 

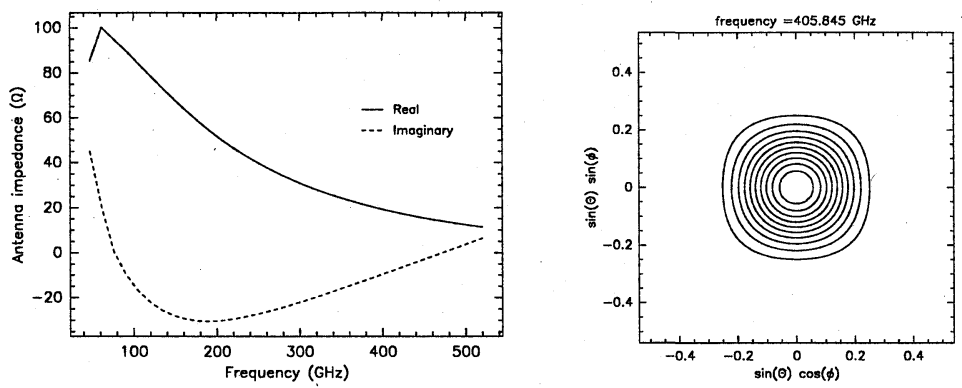

FIGURE 4. On the left: Impedance of an antenna array shown on figure 3. Calculations were done using generalization of method described in [3]. On the right: Beammap of one of $400 \mathrm{GHz}$ pixels. Beammaps of $100 \mathrm{GHz}$ and $200 \mathrm{GHz}$ channels have the same shape.

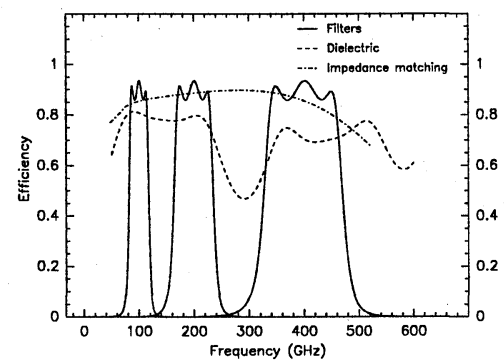

FIGURE 5. Losses due to coupling of real impedance transmission line to antenna array and due to reflections on dielectric interfaces superposed with filters bandpasses. In a case of infinite silicon slab dashed line would be a horizontal equal to $n /(n+1)=0.77$, where $n$ is the silicon refraction index.

30-40\%). To reduce dielectric interface reflections, "layered cake" structure with graded refraction coefficient was used. Silicon wafer with thickness $172 \mu \mathrm{m}$ from the direction of incoming radiation is covered with quartz $216 \mu \mathrm{m}$ thick and $405 \mu \mathrm{m}$ teflon layer.

\section{ACKNOWLEDGEMENTS.}

This research was supported by NASA grant NAG5-10317. Alexey Goldin is supported by NRC Associateship stipendary award.

\section{REFERENCES}

1. Blinchikoff, H. J., and Zverev, A. I., Filtering in the time and frequency domains, Wiley, New York, 1976

2. Ward, J., et al., "Supermix: a Flexible Software Library for High Frequency Circuit Simulation, Including SIS Mixers and Superconducting Elements", in Proceedings, Tenth International Symposium on Space Terahertz Technology, 1999, http://www.submm.caltech.edu/supermix

3. Eleftheriades, G. V., and Rebeiz, G. M., IRMMW (1993) 